\title{
Study and Design of SDN Intelligent Campus Architecture Based on IPv6
}

\author{
Chen Yange ${ }^{1}$, Yang Xiaopin ${ }^{2}$, Zhang Zhili ${ }^{3}$, Zhao Liang $^{4}$ \\ Xuchang University ${ }^{1,3,4}$ \\ Nanchang institute of Technology ${ }^{2}$ \\ chenyange_ok@126.com
}

\begin{abstract}
Against the backdrop of Internet of Things on campus, this paper makes Internet of things cover the entire campus through wired and wireless accesses. As a result, campus sees the fulfillment of information transfer via computers, mobile phones, devices and other articles, which is the embodiment of intelligence. Besides, OPNET is used for modeling and simulation of wireless network structure in SDN intelligent campus, with a view to analyzing the feasibility and advantages of various campus network topologies. Then, a reasonable scheme is presented responding to different types of topologies, which is conducive to better building an intelligent campus.
\end{abstract}

Keywords: Internet of things; intelligent campus; OPNET; SDN

\section{Introduction}

At present, with the rapid development and extensive application of information technology, mobile phones, computers, iPad and digital cameras, college teachers and students have higher demands for information technology, making the development of intelligent campus inevitable. Growing application of information on campus has led to the expansion of intelligent campus and Internet of Things. Moreover, there are increasing needs for IPv6 addresses, because the original IPv4 addresses have been far from meeting the demands of a newly intelligent campus. For colleges, the primary issue is how to build an intelligent campus based on IPv6.

As a significant symbol of comprehensive strength and overall image of school building, intelligent, digitized and information-based campuses emerge as an elemental, long-term and continuous task for colleges to better cultivate talents. Alternatively, an intelligent campus will greatly facilitate the life of teachers and students. Although great achievements have been made in building intelligent campuses at home and abroad, there is still a need for improvement.

Having evolved from digitalized campuses, intelligent campuses, based on Internet of Things, new and high intelligent technologies and Next-generation Network, take full advantage of resources to gather, integrate, process, store, transmit and apply campus information [1]. All this is to make teaching, scientific research, management and services in colleges become intelligent, digitalized and information-based; to reasonably distribute and utilize education resources [2]; and to improve quality of school running, efficiency in school management, scientific research and management levels, thus ensuring the running of school in a rapid, intelligent and harmonious manner.

IPv4 addresses are running out, while there are considerable IPv6 addresses constituting the foundation of intelligent campuses. Meanwhile, in consideration of the growing trend of SDN, this paper researches intelligent campus by combing IPv4/v6 with SDN. SDN intelligent campus architecture is presented, and on that basis, part of stub networks is simulated to lay a solid basis for building reasonable and applicable wisdom network. 


\section{SDN Technology}

Software Defined Network (SDN), new network innovation architecture, has completely open control by following the principles of decoupling control plane from data plane of network devices, developing controller implementation strategies and issuing flow tables. Users can customize any strategies for network routing and transmission rules to be achieved. Flow tables are operated by OpenFlow repeater [3, 4], thence realizing flexible control and intelligent management of network traffic [5].

SDN can be defined in a broad and narrow sense. Broadly, SDN means that upper-layer applications open resources to create various network architectures of software programming control [10]; narrowly, it refers to SDN implemented based on OpenFlow protocol. SDN realized a great leap from experimental technology to network deployment in 2012, when operators like Deutsche Telekom began to develop and deploy SDN [6] and other countries were also engaged in research and deployment. However, SDN for basic architecture of NGI, in particular effectively integrating and applying IPv6 and $\mathrm{SDN}$, remains to be further researched.

\section{Overall SDN Architecture for Intelligent Campus Based on IPv6}

Topologies for traditional campus networks are generally three-layer network architecture. With the development of science and technology, wireless network has gradually covered the entire campus. Upon improvements, wireless signals are sent to wired network via WIFI wireless access points (APs). In this way, teachers and students can have access to the Internet anywhere at any time, which embodies intelligent design. Meanwhile, stubs are used to establish ZigBee network and transmit to the monitoring center, for the purpose of facilitating management and monitoring of devices and objects on campus. Additionally, mobile network is integrated with WIFI. When intelligent campus undergoes SDN improvements, there is no need to deploy each routing node as long as network rules are defined in use as needed. Figure 1 shows the improved network topology.

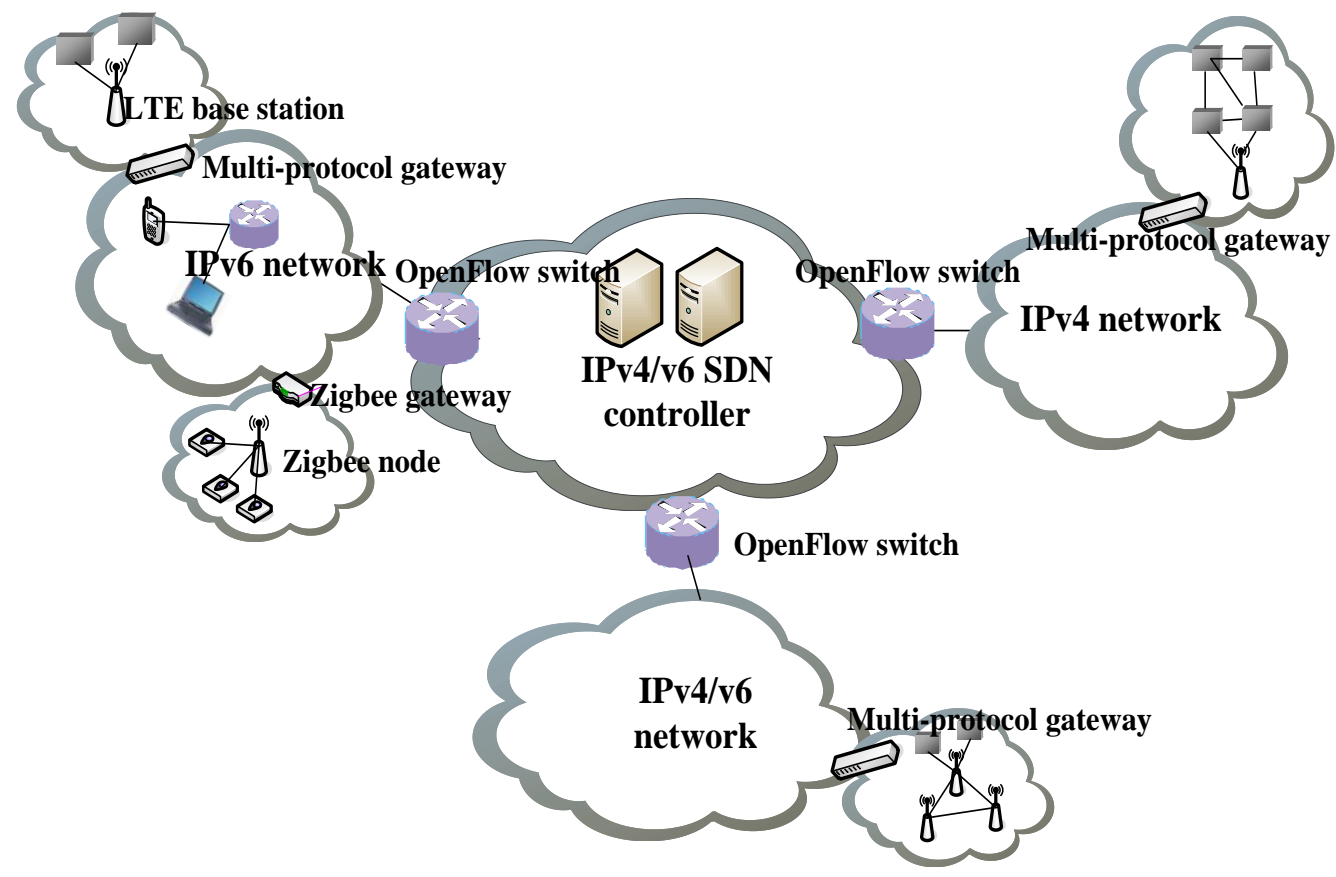

Figure 1. SDN Intelligent Campus Architecture Based on IPv6 
As for intelligent campus network, lightweight IPv6 is adapted to standard IPv6 and multi-protocol gateways are deployed. To this end, the core convergence layer employs SDN-based IPv4/IPv6 network to operate such IPv6 transition technologies as protocol translation and DS-Lite in the form of software. Besides, address resources are managed as a whole. OpenFlow switch of network devices uses OpenFlow protocol, to which new flow tables can be added, for instance, IPv6 message headers can be added to IPv4 data packages and vice versa [7]. In turn, such additional message headers can be gotten rid of in the process of decapsulation, making it possible to identify the protocol traversing networks. OpenFlow switch accounts for data forwarding, which can separate not only control plane from data plane but Protocol Translation from data forwarding.

On the stub network, Internet of Things nodes have access to network wirelessly via a multi-protocol gateway, while Internet of Things access network communicate with a multi-protocol gateway via wireless or wired networks. In future IPv6 core network communications, network layer uses IPv6 protocol. When RPL and 6LoWPAN [8] protocols are not supported in routes of low-power network, application layer uses CoAP [9] protocol.

Various networks are allowed to access the IPv4/v6 protocol-based SDN controller through different access technologies, which can realize interconnection between different types of networks, including fixed and mobile networks.

\section{Detailed Design of Intelligent Campuses}

\subsection{Campus All-purpose Card}

Smart campus card has basically taken shape, with gradually mature application system and technical support. Many colleges and universities have established a perfect campus all-purpose card system that introduces RFID technology. A RFID system comprises three parts: RF reader, RF electronic tag and centralized information system. The electronic tag sends information to centralized information system for real-time processing.

Figure 2 shows the composition of RFID subnet of all-purpose card. Campus allpurpose cards are used in supermarkets, water rooms, restaurants and libraries. Generally, wired network is used for connection, but wireless network is used in dormitory doors. 


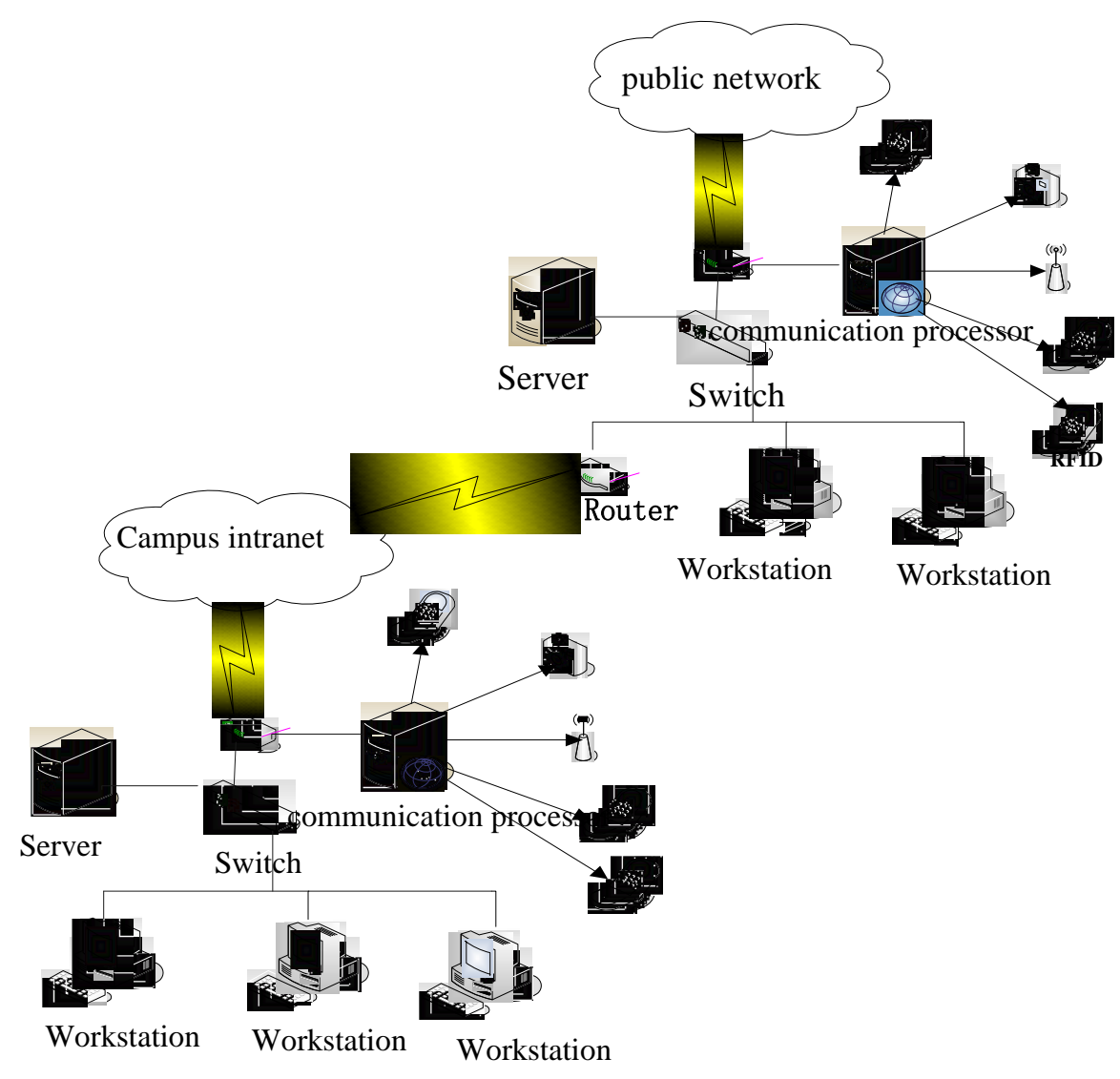

Figure 2. RFID Subnet of All-purpose Card

\subsection{Intelligent Classroom}

Intelligent classrooms should include intelligent tutoring, attendance of teachers and students, curtain control, lighting control, temperature control, video surveillance and remote control. Peer-to-peer network structure is suitable for passing information among students in classrooms, while network infrastructure is applied to monitor sensor nodes ${ }^{[2]}$. The organizational chart is shown in Figure 3.

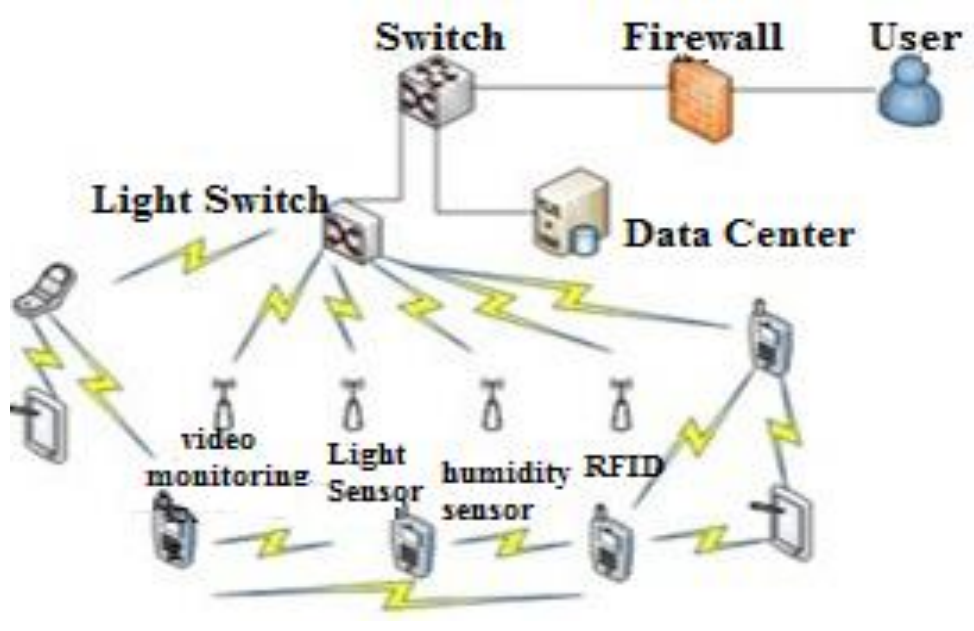

Figure 3. Subnet of Intelligent Classroom 
In terms of intelligent classroom control, curtain and lighting control gather information through illumination sensor nodes; air-conditioning and fan control are realized through temperature sensor nodes; video surveillance system monitors students' learning; and apparatus failure detection module accounts for receiving failure information sent by other modules and keeping records. Moreover, information-based device management applies Internet of Things to campus devices for tracking and locating to facilitate management. Intelligent multi-media devices, lamplight and curtains in classroom can be automatically adjusted as needed, so as to optimize the learning environment. Each classroom is equipped with an enterprise-like punch-card machine for people counting, and then the gathered data is stored in the server for later evaluation. Data sending and receiving makes it possible for interaction between teachers and students.

\subsection{Campus Security}

Campus security system needs to provide such services as fire prevention, entrance guard and guard against theft. Access control for classrooms is to set time for automatic locking and increase persons allowed in. Such devices as card reader, camera, electronic lock and fingerprint reader are installed at the entrance of the machine room, so personnel concerned cannot be allowed in until they pass the validation process. Besides, video surveillance can collect images, sounds and alerting signals through cameras. When it comes to fire alarm, heat sensors are used not only to see whether temperature in laboratory gets too high or not, but to keep real-time records of temperature data which are output into webpage.

ZigBee supports the dynamic generation of network by nodes. Accordingly, combing real-time network, ZigBee protocol is employed to establish wireless sensor network for security monitoring of campus environment. The surveillance center receives signals from sensors via wireless routers and GPRS wireless network connection. In case of fires, moving students with a ZigBee module can be found the real-time location, which brings convenience to rescue and saves rescue time, hence ensuring the life safety of students.

\subsection{Fusion of Intelligent Stubs}

Intelligent campus introduces ZigBee campus gateway devices to integrate ZigBee network with communication network on the Internet of Things and exercise remote network and mobile phone control. GSM module is built in through system bus, so that students can establish correspondence to remote mobile devices using GMS mobile phone network and inform managers of special cases on campus by sending short messages. ZigBee module can be embedded in students' mobile phones. If a student is in danger, managers can obtain his or her accurate position for rescue, thus implementing such functions as mobile phone location, remote control and mobile control.

To integrate stub network with campus network on the Internet of Things, the ZigBee gateway offers Ethernet interface or built-in wireless network card (supporting IEEE 802.11), which can connect computer terminal and campus network. In this way, even though managers are on business, they can implement remote control via a computer, as shown in Figure 4. 


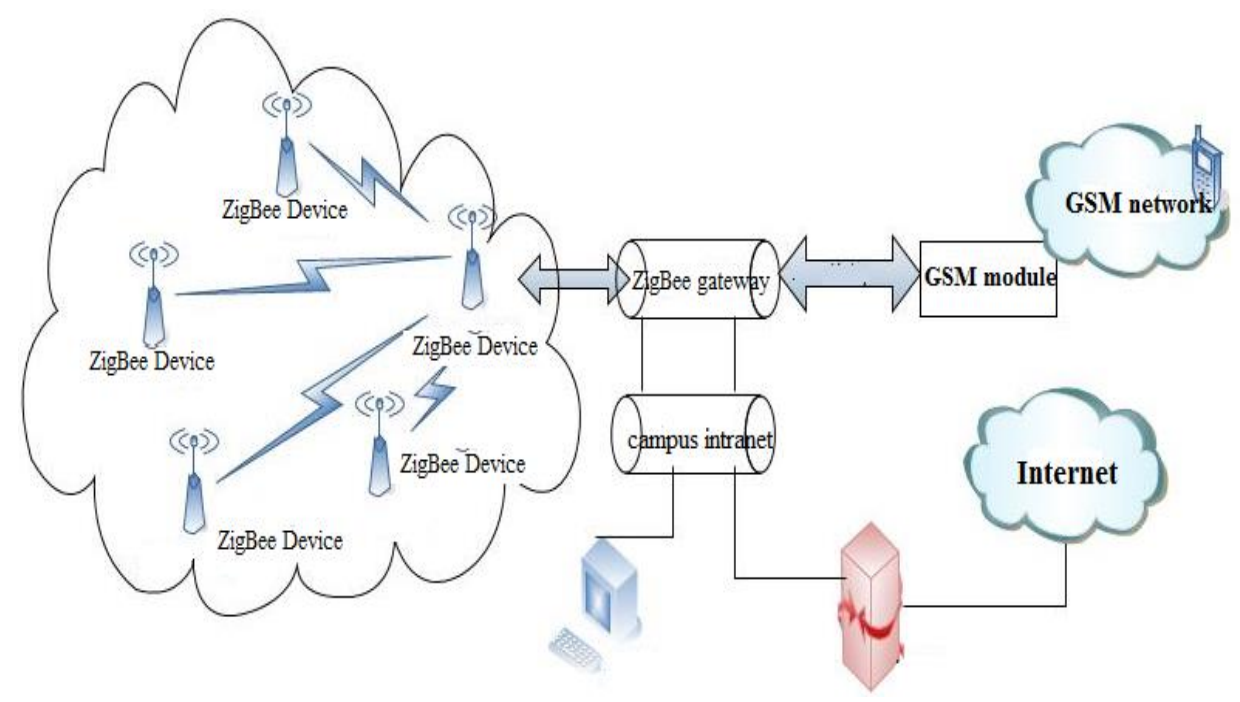

Figure 4. Integration of ZigBee Campus Network, Mobile Phone Network and the Internet

Integration of campus WIFI sand LTE network can be fulfilled through RF-photon fusion system. Furthermore, RF switching and radio-over-fiber technologies can be used to realize fiber distribution of mixed signals like LET and WIFI. Multiple APs are integrated. RF switching module can ensure that LTE signals of each AP and communication module radiate from a remote antenna, as shown in Figure 5.

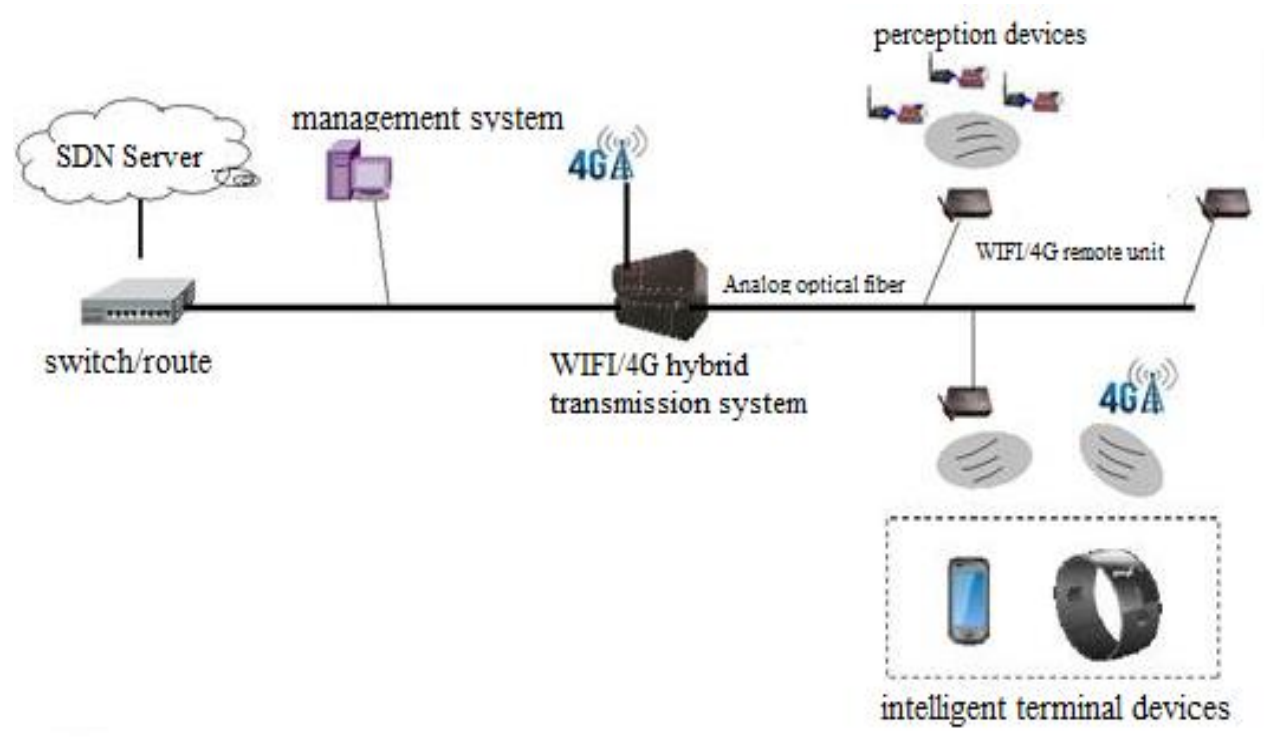

Figure 5. Mobile Internet Network Based on RF-photon Fusion System

\section{Simulation Design}

In consideration of limitations, we simulate ZigBee and mobile networks using OPNET. The following describes the simulation results:

As can be seen from simulation results in Figure 6 and 7, ZigBee network selects mesh topology with less loads and network delays than other networks. 




Figure 6. Delay Simulation of Mesh Topology

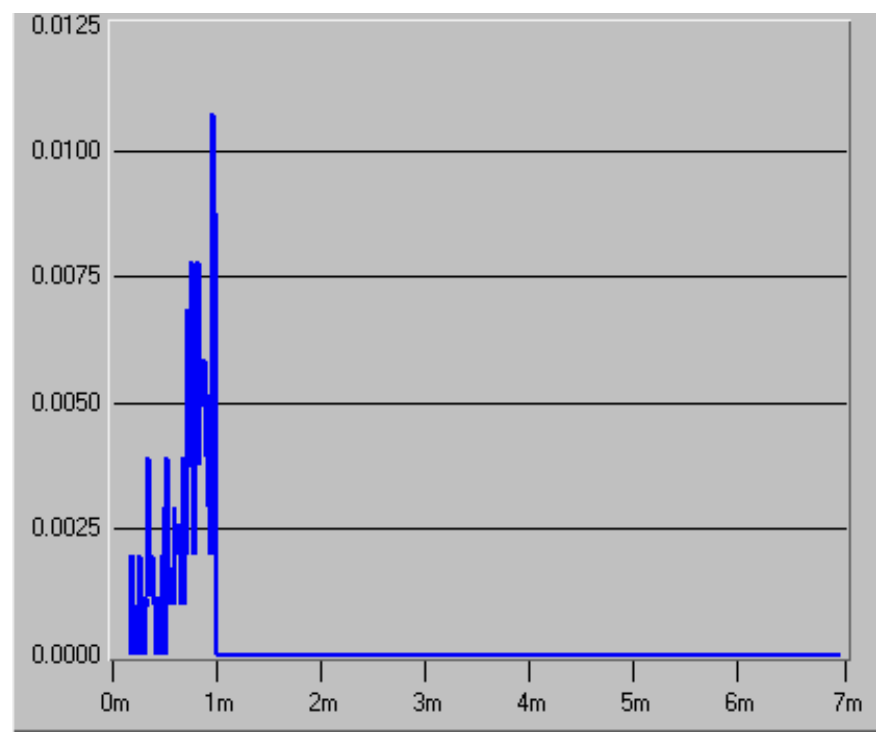

Figure 7. Load Simulation of Mesh Topology

As can be seen from simulation results of bit error rate and network throughput of $1 \mathrm{M}$ and 10M bandwidth in Figure 8, appropriately increasing bandwidth can increase network throughput and improve network utilization. 


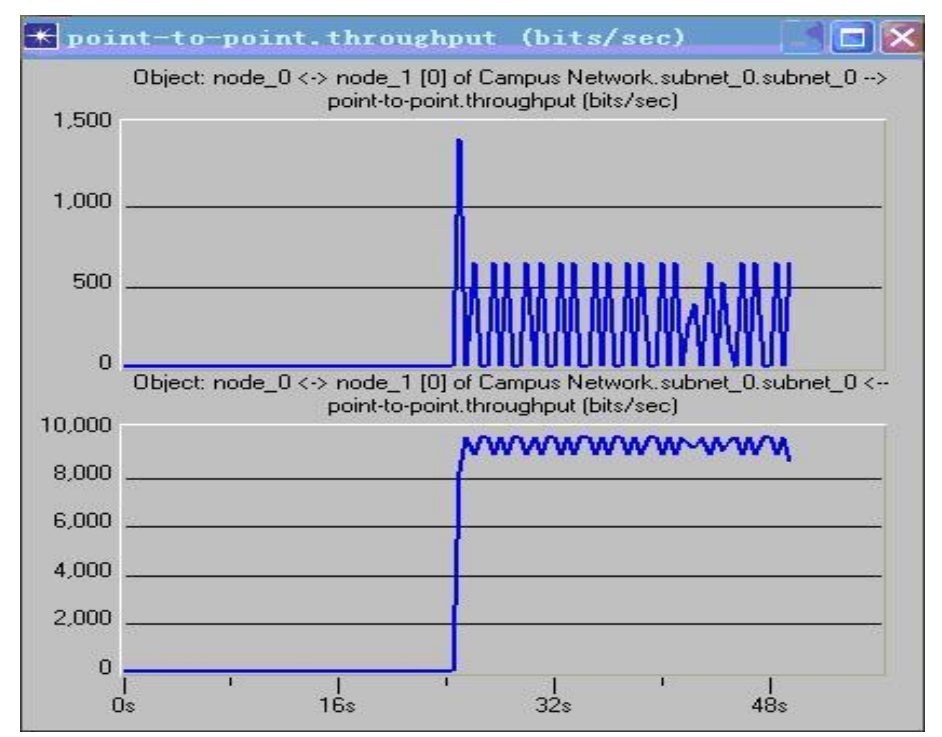

\section{Figure 8. Comparison of Network Throughput at 1M and 10M Bandwidth}

A simulation is made on mobile nodes for changes in network services. Internet connection is firstly observed. Then, an analysis found that each node fluctuates greatly at the beginning and network traffic seems to be stable after some time, as shown in Figure 9.



Figure 9. Network Continuity

After a series of simulations, a conclusion can be drawn that simulating wireless and mobile networks is conducive to finding more appropriate ways to build SDN intelligent campus network based on IPv6.

\section{Acknowledgements}

Grant funds from four projects are highly appreciated. The projects have the next generation network technology innovation project of ministry of education (NGII20150610), Science and Technology project of Education Department of Henan Province (15A510012 、17A520055), Science and Technology project of Education Department of Jiangxi Province (GJJ151131). 


\section{References}

[1] Y. W. Cui, "Smart campus construction in the era of smart phones", Chinese market, vol. 23, no. 3, (2015).

[2] H. R. Feng, "Construction of intelligent campus of Xuchang University", Combined comparison database of College Students, vol. 5, (2014).

[3] Q. Sun and C. F. Xie, "Design and implementation of IPv6 Transition Technology Based on SDN architecture", Telecommunication Science, vol. 4, (2014).

[4] S. Arlimatti, S. Hassan and A. Habbal, "Minimization of Communication Cost between Controller Domains with Graph Partitioning Algorithms", Revista de la Facultad de Ingeniería, vol. 31, no. 5, (2016), pp. 01-08.

[5] S. N. Hu, "Application of new neural network technology in traffic volume prediction", Revista de la Facultad de Ingeniería, vol. 31, no. 6, (2016), pp. 75-83

[6] J. Huang, "Analysis of the application prospect of SDN technology in MAN", Jiangsu communication, vol. 2, (2016).

[7] S. J. Ma and D. Y. Hu, "Implementation of dynamic traffic control for multi service based on IP fusion switching technology based on SDN", Science in China: Information Science, vol. 46, no. 5, (2016).

[8] S. N. Tang, "Research and implementation of lightweight IPv6 network fusion model", Degree thesis of Nanjing University of Posts and Telecommunications, vol. 3, (2013).

[9] S. Z Sensinode, K. Hartke and C. Bormann, "Constrained Application Protocol (CoAP) draft-ietf-corecoap-04", https://datatracker.ietf.org/doc/draft-ietf-core-coap/, (2011).

[10] L. Guo and J. H. Wang, "The impact of the evaluation of IP devices on the evolution of SDN network architecture", Paper compilation on 2015 annual conference of China communications Institute, information and communication network technology committee, (2015).

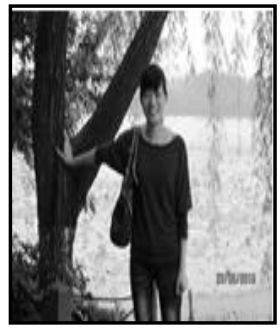

\section{Authors}

Chen Yange, she graduated from Henan Polytechnic University, and is a lecturer in College of Information Engineering, Xuchang University, Henan Province, where she is teaching wireless communication network and network courses. Her area of research is the next generation Internet, SoftwareDefined Networking (SDN), Internet of things.

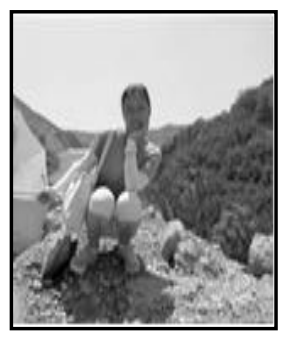

Yang Xiaopin, she graduated from Wuhan University, and is a lecturer in Nanchang institute of technology, JiangXi Province, where she is teaching power electronic, integrated automation and engineering economy. Her area of research is modeling and simulating, wind power, and power electronic.

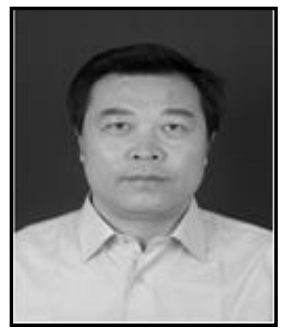

Zhang Zhili, he graduated from South China University of Technology, and is a Professor in College of Information Engineering, Xuchang University, Henan Province, where he is teaching network courses. His area of research is the next generation Internet, Software-Defined Networking (SDN). 
International Journal of Future Generation Communication and Networking Vol. 9, No. 12 (2016) 